\title{
Growing Neural Gas - A Parallel Approach
}

\author{
Lukáš Vojáček ${ }^{1}$ and Jiří Dvorský \\ 1 IT4Innovations Centre of Excellence \\ Ostrava, Czech Republic \\ lukas.vojacek@vsb.cz \\ 2 Department of Computer Science, \\ VŠB - Technical University of Ostrava, 17. listopadu 15, \\ 70833 Ostrava, Czech Republic \\ jiri.dvorsky@vsb.cz
}

\begin{abstract}
The paper deals with the high dimensional data clustering problem. One possible way to cluster this kind of data is based on Artificial Neural Networks (ANN) such as SOM or Growing Neural Gas (GNG). The learning phase of the ANN, which is time-consuming especially for large high-dimensional datasets, is the main drawback of this approach to data clustering. The parallel modification, Growing Neural Gas, and its implementation on the HPC cluster is presented in the paper. Some experimental results are also presented.
\end{abstract}

Keywords: growing neural gas, high-dimensional dataset, high performance computing.

\section{Introduction}

Recently, the issue of high-dimensional data clustering has arisen together with the development of information and communication technologies which support growing opportunities to process large data collections. High dimensional data collections are commonly available in areas like medicine, biology, information retrieval, web analysis, social network analysis, image processing, financial transaction analysis and many others.

Two main challenges should be solved to process high-dimensional data collections. One of the problems is the fast growth of computational complexity with respect to growing data dimensionality. The second one is specific similarity mea-surement in a high-dimensional space. As presented in [1], Beyer et al. for any point in a high-dimensional space the expected distance, computed by Euclidean measure to the closest and to the farthest point, shrinks with growing dimensionality. These two reasons reduce the effectiveness of clustering algorithms on the above-mentioned high-dimensional data collections in many real applications.

The authors propose an effective data clustering algorithm which is based on Growing Neural Gas (GNG) 7]. The computational complexity is resolved by the parallel implementation of GNG. Some technical problems have to be resolved in 
order to effectively train such kind of neural network using an High Performance Computing (HPC) cluster with MPI. The traditional serial approach to training GNG is also considered in the paper. The serial learning GNG algorithm is used for benchmarking the parallel version of GNG. In other words, parallel GNG has to produce the same network and should be an order of magnitude faster in the ideal case.

\section{Artificial Neural Networks}

\subsection{Self Organizing Maps}

Self Organizing Maps (SOM), also known as Kohonen maps, were proposed by Teuvo Kohonen in 1982 [4. SOM is a kind of artificial neural network that is trained by unsupervised learning. Using SOM, the input space of training samples can be represented in a lower-dimensional (often two-dimensional) space [5], called map. SSuch model is efficient in structure visualization due to its feature of topological preservation using a neighbourhood function.

SOM consists of two layers of neurons (see Fig. 1): an input layer that receives and transmits the input information and an output layer, the map that represents the output characteristics. The output layer is commonly organized as a two-dimensional rectangular grid of nodes, where each node corresponds to one neuron. Both layers are feed-forward connected. Each neuron in the input layer is connected to each neuron in the output layer. A real number, or weight, is assigned to each of these connections.

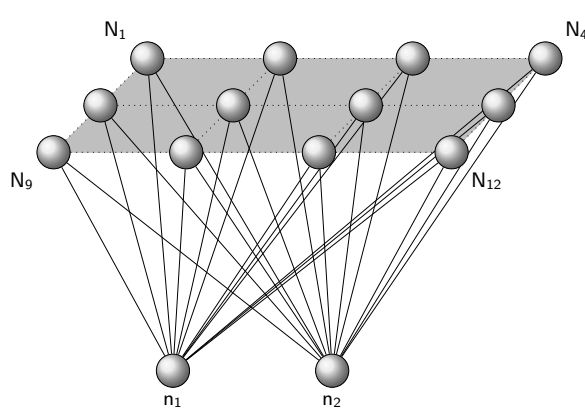

(a) Global view on SOM structure

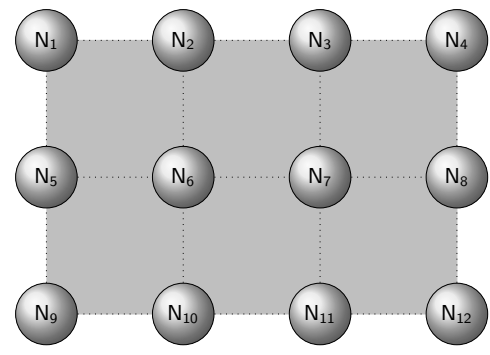

(b) SOM output layer

Fig. 1. Basic Schema of SOM

\subsection{Growing Neural Gas}

The principle of this neural network is an undirected graph which need not be continuous. Generally, there are no restrictions on the topology. The graph is generated and continuously updated by competitive Hebbian Learning [6 9]. 
According to the pre-set conditions, new neurons are automatically added and connections between neurons are subject to time and can be removed. GNG can be used for vector quantization by finding the code-vectors in clusters 3], biologically influenced [10], image compression, disease diagnosis.

GNG works by modifying the graph, where the operations are the addition and removal of neurons and edges between neurons. An example of the operation is shown in Figure 2

To understand the functioning of GNG, it is necessary to define the algorithm. The algorithm described by Algorithm 1 is based on the original algorithm [2] [3], but it is modified for better continuity in the SOM algorithm. The description of the algorithm has been divided for convenience into two parts. In the first part of Algorithm 1 the overall functionality is described. The second part of Algorithm 2 describes one iteration, which means the descriptions of variables without dependence on time.

Remark The notation used in the paper is briefly listed in Table 1

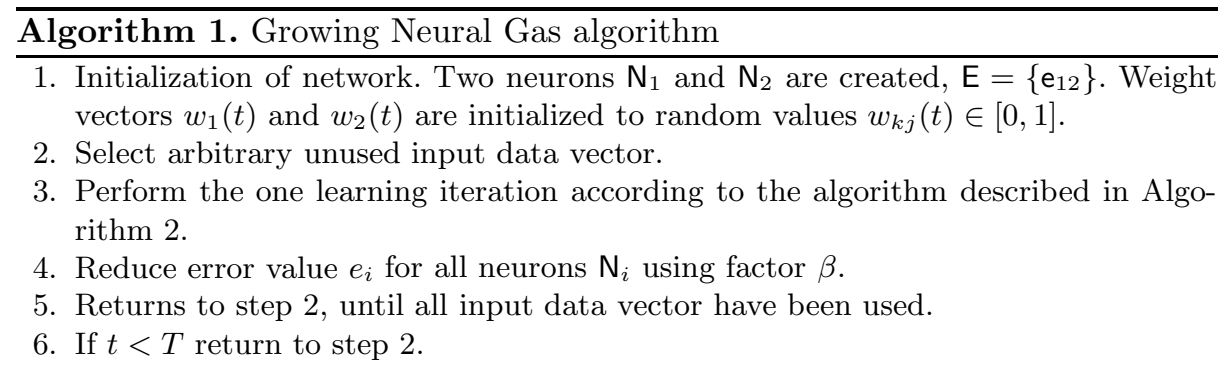

\section{Parallelization}

Parallelization focuses on the equally distribution of neurons, where new neurons are allocated to the process with the lowest number of neurons. The advantage of this distribution is constant workload processes. The disadvantage is increase communication between processes.

After analysing the GNG learning algorithm we identified the one most processor time-consuming area. This part was selected as a candidate for the possible parallelization. The selected area is:

Finding BMU - this part of GNG learning can be significantly accelerated by dividing the GNG output layer into smaller pieces. Each piece is then assigned to an individual computation process. The calculation of the Euclidean distance among the individual input vector and all the weight vectors to find BMU in a given part of the GNG output layer is the crucial point of this part of GNG learning. Each process finds its own, partial, BMU in its part of the GNG output layer. Each partial BMU is then compared with 
Table 1. Notation used in the paper

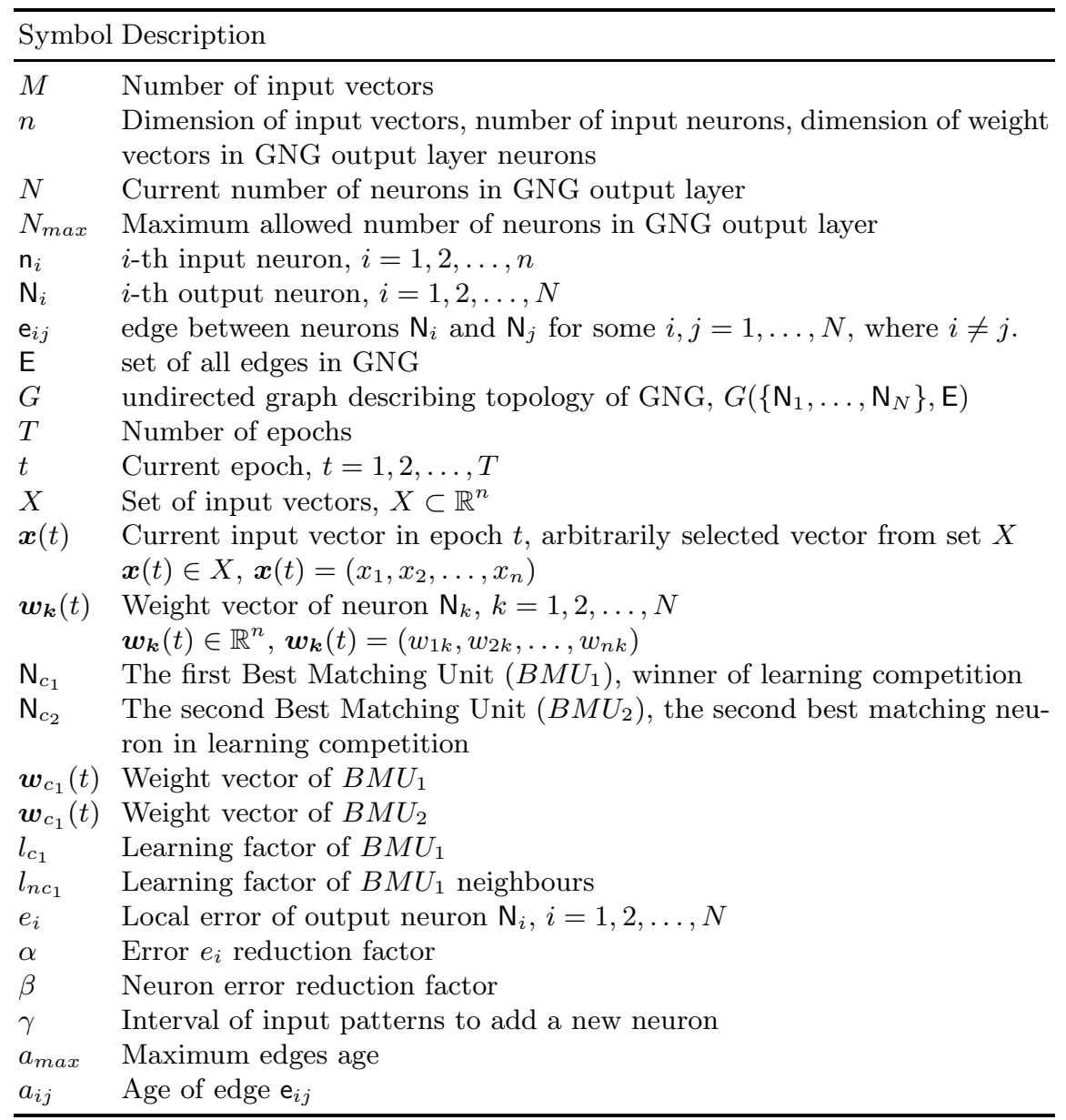




\section{Algorithm 2. One iteration of the Growing Neural Gas algorithm}

1. Find neurons BMUs neurons $\mathrm{N}_{c_{1}}$ and $\mathrm{N}_{c_{2}}$.

2. Update the local error $e_{c_{1}}$ of neuron $\mathrm{N}_{c_{1}}$

$$
e_{c_{1}}=e_{c_{1}}+\left\|\boldsymbol{w}_{c_{1}}-\boldsymbol{x}\right\|^{2}
$$

3. Update the weight vector $\boldsymbol{w}_{c_{1}}$ of neuron $\mathbf{N}_{c_{1}}$

$$
\boldsymbol{w}_{c_{1}}=\boldsymbol{w}_{c_{1}}+\boldsymbol{l}_{c_{1}}\left(\boldsymbol{x}-\boldsymbol{w}_{c_{1}}\right)
$$

4. For all neurons $\mathrm{N}_{k}$ where exists edge $\mathrm{e}_{c_{1} k}\left(\mathrm{~N}_{c_{1}}\right.$ neighbourhood)

(a) Update the weights $\boldsymbol{w}_{k}$ using $l_{n c_{1}}$ learning factor

$$
\boldsymbol{w}_{k}=\boldsymbol{w}_{k}+l_{n c_{1}}\left(\boldsymbol{x}-\boldsymbol{w}_{\boldsymbol{k}}\right)
$$

(b) Increase age $a_{k c_{1}}$ of edge $\mathrm{e}_{c_{1} k}$

$$
a_{k c_{1}}=a_{k c_{1}}+1
$$

5. If there is no edge between neurons $\mathrm{N}_{c_{1}}$ and $\mathrm{N}_{c_{2}}$, then create such edge. If the edge exists, the age is set to 0 .

6. If any edge has reached the age of $a_{\max }$, it is removed.

7. If there is a neuron without connection to any edge, the neuron is then removed.

8. If the number of processed input vectors in the current iteration has reached the whole multiple of the value $\gamma$ and the maximum allowed number of output neurons is not reached, add a new neuron $\mathrm{N}_{N+1}$. The location and error of the new neuron is determined by the following rules:

(a) Found neuron $\mathrm{N}_{b}(\mathrm{NBE})$ which has the biggest error $e_{b}$.

(b) Found neuron $\mathrm{N}_{c}(\mathrm{NSE})$ among neighbours of neuron $\mathrm{N}_{b}$ and has the biggest error $e_{c}$ among these neighbours.

(c) Create a new neuron $\mathrm{N}_{N+1}$ and the value of $w_{n}$ is set as:

$$
\boldsymbol{w}_{N+1}=\frac{1}{2}\left(\boldsymbol{w}_{b}+\boldsymbol{w}_{c}\right)
$$

(d) Creating edges between neurons $\mathrm{N}_{b}$ and $\mathrm{N}_{N+1}$, and also between neurons $\mathrm{N}_{c}$ and $\mathrm{N}_{N+1}$.

(e) Removed edge between neurons $\mathrm{N}_{b}$ and $\mathrm{N}_{c}$.

(f) Reduction of error value in neurons $\mathrm{N}_{b}$ and $\mathrm{N}_{c}$ using the multiplying factor $\alpha$. Error for neuron $\mathrm{N}_{N+1}$ is equal to the new error of neuron $\mathrm{N}_{b}$. 


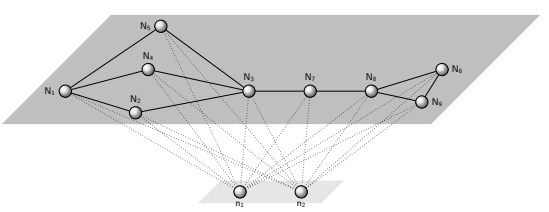

(a) Original condition

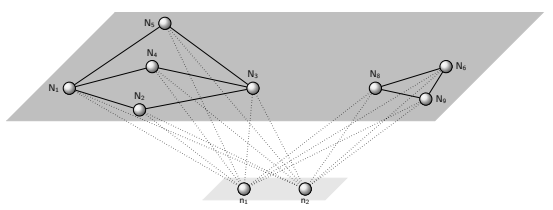

(c) Removal of edges between $N_{7}$ a $N_{8}$, (d) Added neuron $N_{10}$ between $N_{5}$ a $N_{3}$ remove neuron $\mathrm{N}_{7}$

Fig. 2. GNG examples

other BMUs obtained by other processes. Information about the BMU of the whole network is then transmitted to all the processes to perform the updates of the BMU neighbourhood.

A detailed description of our approach to the parallelization process is described in Fig. 3 ,

The parallelization of GNG learning was performed on an HPC cluster, using Message Passing Interface (MPI) technology. MPI technology is based on effective communication between processes. That means that one application can run on many cores. The application uses MPI processes which run on individual cores. The processes are able to send and receive messages and data, communicate etc. Detailed information about HPC and MPI technology is provided, for example, in [8].

\subsection{Description of Proposed Approach}

This subsection includes a detailed description of our approach. Initially, each process reads the training input vectors and on the first process are create two neurons. During the calculation, new neurons are equally distributed on the processes. We do not store the complete GNG graph, we only store parts of it in the appropriate computational nodes.

This approach results in the following significant advantage: neurons are equally distributed among the processes corresponding to the used cores, contrary to the sequential approach, where the whole graph is allocated to only one core (and where there may not be enough memory). For a more precise

\footnotetext{
${ }^{1}$ A specification of MPI is available on the web: http://www.mpi-forum.org/ The functions used in the experiment with a link to MPI.Net are available on the web: http://osl.iu.edu/research/mpi.net
} 


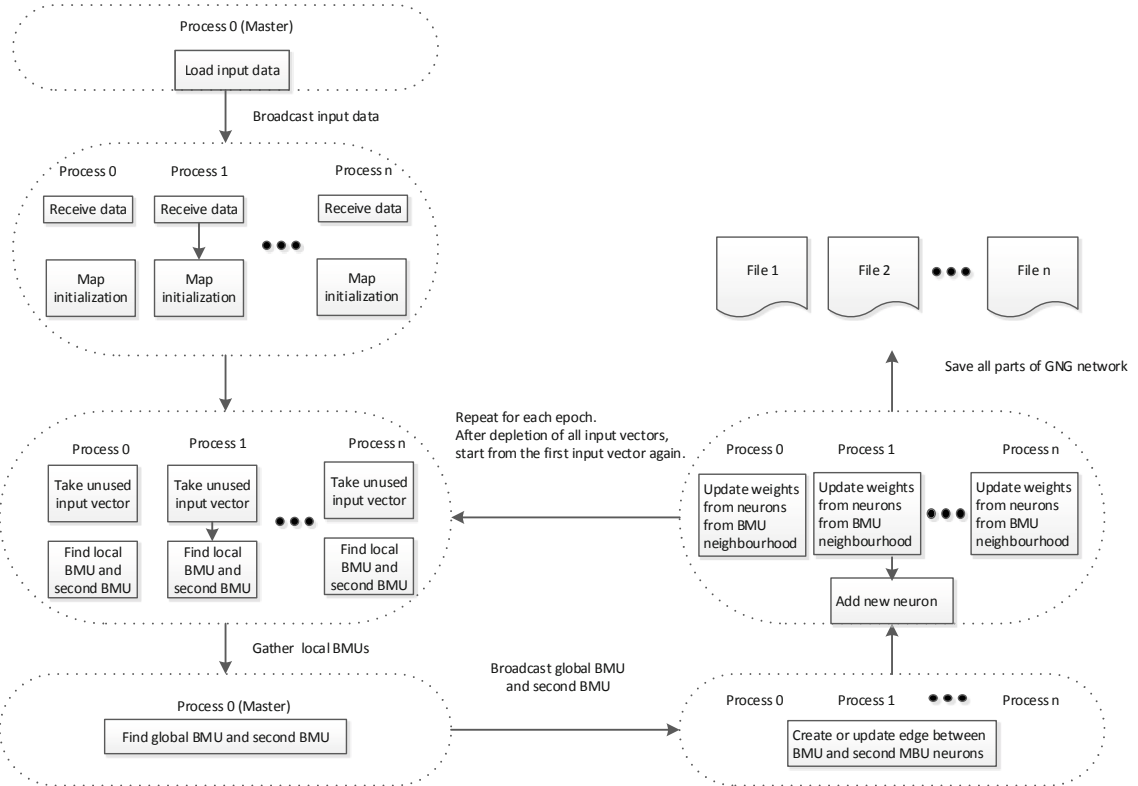

Fig. 3. Parallel Algorithm

illustration: consider having three computation servers, each with $16 \mathrm{~GB}$ of memory. If we use the sequential GNG version, we can only use $16 \mathrm{~GB}$ of memory on one server. But in the case of the parallel SOM version, we can use all the memory, all 48GB. (The graph can be up to three times larger.)

In the main computational phase, one BMU and a second BMU are founded for each input vector. Thus, each processor needs to compute its local BMU and second BMU within its neurons, after which, each local BMU and second BMU (and their position in the network) are shifted onto one process using the MPI function GatherFlattened to determine the global BMU and second BMU. It is possible to use another MPI functions as well, which can provide this selection at one time, but after testing we have found that the experiments took much more time than our presented approach. A global winning BMU and second BMU are then distributed using the MPI function Broadcast on all the processes. Now if there is a edge between the first BMU and the second BMU then age is set to zero otherwise creates edge between this two neurons. Next, the neighbourhood of the BMU in each process is known and, consequently, the weights of the neurons matching are actualized. If the condition is met for adding a new neuron, the process with the lowest number of neurons add a new neuron. A detailed description of adding a new neuron can be found in Fig. 4. This procedure is repeated until all the input training vectors are exhausted (until we have finished one epoch). 


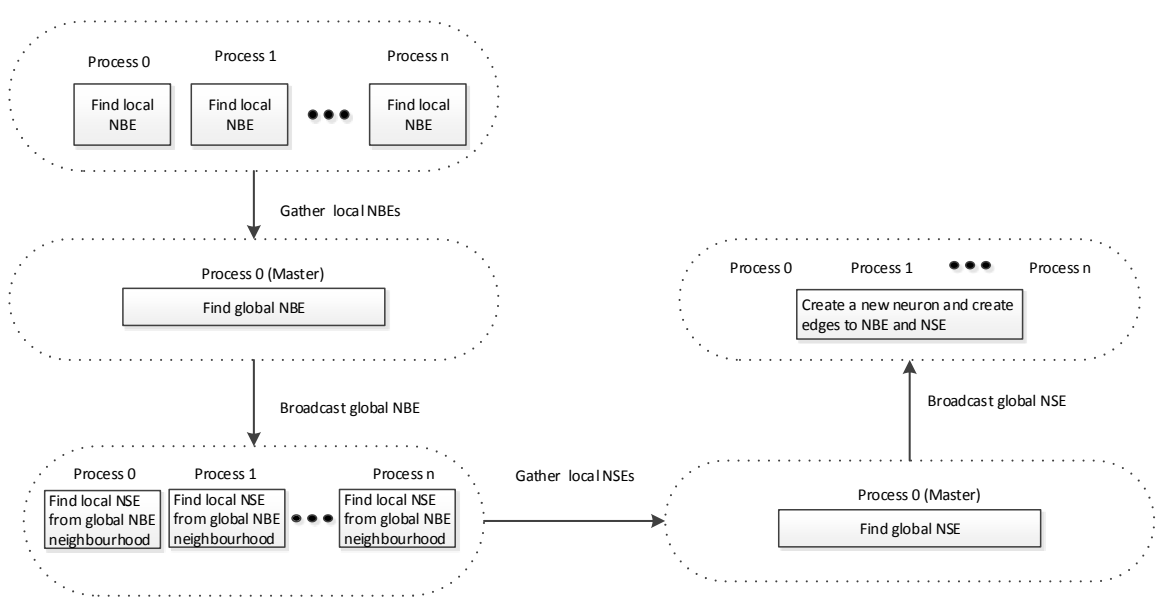

Fig. 4. Parallel Algorithm for adding a neuron

\section{Experiments}

\subsection{Experimental Datasets and Hardware}

Two datasets were used in the experiments. The first dataset was commonly used in Information Retrieval - Medlars. The second one was the test data for the elementary benchmark for clustering algorithms[11.

Medlars Dataset. The Medlars dataset consisted of 1,033 English abstracts from a medical science 2 . The 8,567 distinct terms were extracted from the Medlars dataset. Each term represents a potential dimension in the input vector space. The term's level of significance (weight) in a particular document represents a value of the component of the input vector. Finally, the input vector space has a dimension of 8,707, and 1,033 input vectors were extracted from the dataset.

Clustering Dataset. Three training data collections called TwoDiamonds, Lsun and Hepta from the Fundamental Clustering Problems Suite (FCPS) are used. A short description of the selected dataset used in our experiments is given in Table 2 .

Experimental Hardware All the experiments were performed on a Windows HPC server 2008 with 6 computing nodes, where each node had 8 processors with $12 \mathrm{~GB}$ of memory. The processors in nodes were Intel Xeon $2.27 \mathrm{GHz}$.

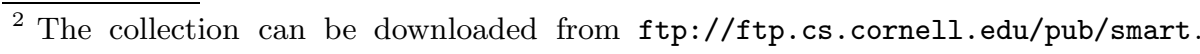

The total size of the dataset is approximately 1.03 MB. 
Table 2. Fundamental Clustering Problems Suite - selected datasets

\begin{tabular}{lcccl}
\hline Name & Cases & \#Vars & \#Clusters & Main Clustering Problem \\
\hline Target & 770 & 2 & 6 & outlying clusters \\
Lsun & 400 & 2 & 3 & different variances in clusters \\
TwoDiamonds & 800 & 2 & 2 & touching clusters \\
\hline
\end{tabular}

The topology with the connection between the head node and computing nodes can be found on the web3 (topology number four). The Enterprise and Private Networks link speed was $1 \mathrm{Gbps}$, the Application link speed was $20 \mathrm{Gbps}$.

\subsection{First Part of the Experiment}

The first part of the experiment was oriented towards a comparison of the standard GNG algorithm and parallel approach to this GNG learning algorithm. The Medlars dataset was used for the experiment. A parallel version of the learning algorithm was run using 2, 8, 16, 24 and 32 MPI processes. The records with an asterisk $\left(^{*}\right)$ represents the results for only one process i.e. this is the original serial learning algorithm and there is no network communication.

GNG parameters are the same for all experiments and are as follows $\gamma=200$, $e_{w}=0.05, e_{n}=0.006, \alpha=0.5, \beta=0.0005, a_{\max }=88, \mathrm{M}=1000, \delta=100$. The achieved computing time is presented in Table 3 .

Table 3. Computing Time with Respect to Number of Cores, Standard GNG Algorithm, Dataset Medlars

\begin{tabular}{cc}
\hline Cores & Computing Time [hh:mm:ss] \\
\hline $1^{*}$ & $00: 35: 41$ \\
2 & $00: 17: 50$ \\
8 & $00: 04: 47$ \\
12 & $00: 03: 56$ \\
16 & $00: 03: 09$ \\
24 & $00: 02: 45$ \\
32 & $00: 02: 32$ \\
\hline
\end{tabular}

As we can see from Table 3, the computing time depends on the number of used cores as well. With a growing number of processors, the computation effectiveness increases, and the computational time is sufficiently reduced.

\subsection{Second Part of the Experiment}

The second part of the experiments was oriented towards comparing the results obtained by the parallel and standard GNG algorithm. The Clustering dataset

\footnotetext{
3 http://technet.microsoft.com/en-us/library/cc719008(v=ws.10).aspx
} 
was used for the experiment. The parallel version of the learning algorithm was run using 16 MPI processes.

GNG parameters are similar to the previous experiment. There are two changes $\mathrm{M}=500$ and $\delta=25$.

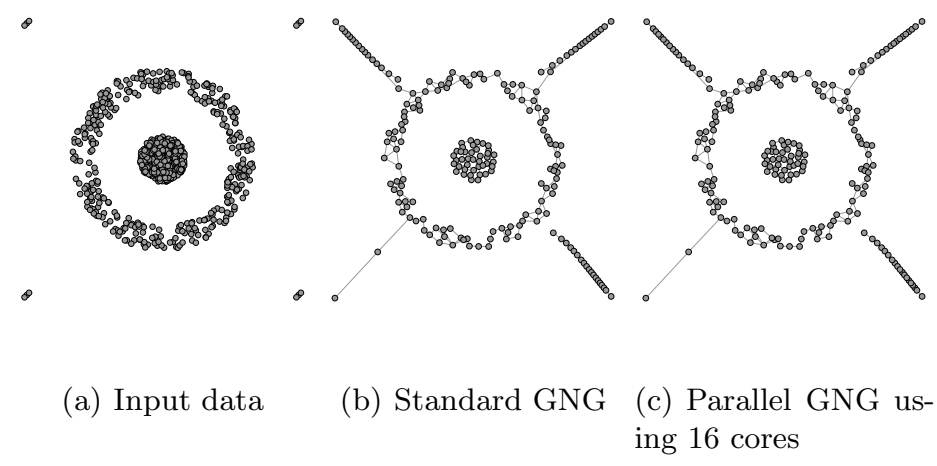

Fig. 5. Results of dataset Target

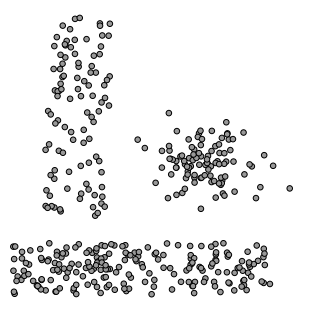

(a) Input data

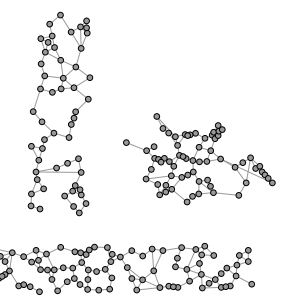

(b) Standard GNG

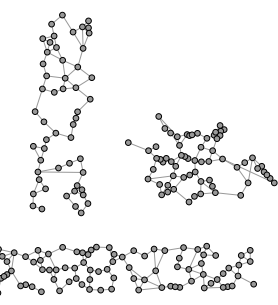

(c) Parallel GNG using 16 cores

Fig. 6. Results of dataset Lsun

In Figures 5(a), 6(a) and 7(a) there are a layout view input data, which are used for training GNG. Outputs of standard GNG algorithm are in Figures 5(b) $6(\mathrm{~b})$ and $7(\mathrm{~b})$, which are the same as in the parallel version. i.e. both versions produce the same network. 


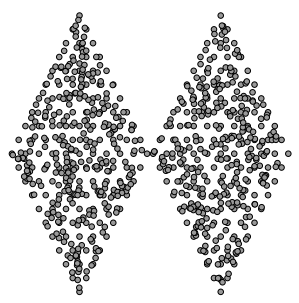

(a) Input data

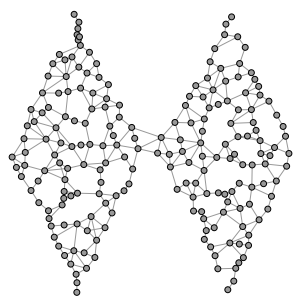

(b) Standard GNG

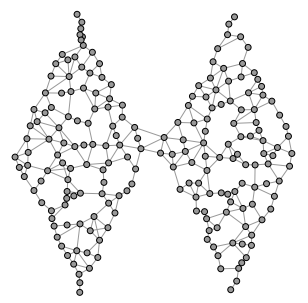

(c) Parallel GNG using 16 cores

Fig. 7. Results of dataset TwoDiamonds

\section{Conclusion}

In this paper the parallel implementation of the GNG neural network algorithm is presented. The achieved speed-up was very good and the results from the standard and parallel version of GNG are same. So we can say that the operation of the parallel version is correct. However, the effectiveness of a parallel solution is dependent on the division of the output layer. An improper division may cause the communication between processes to be very time consuming.

In future work we intend to focus on the sparse date, use combinations of neural networks for improved result and improved acceleration.

Acknowledgement. This work was supported by the European Regional Development Fund in the IT4Innovations Centre of Excellence project (CZ.1.05/1.1.00/02.0070) and by the Development of human resources in research and development of latest soft computing methods and their application in practice project, reg. no. CZ.1.07/2.3.00/20.0072 funded by Operational Programme Education for Competitiveness, co-financed by ESF and state budget of the Czech Republic.

\section{References}

1. Beyer, K., Goldstein, J., Ramakrishnan, R., Shaft, U.: When is nearest neighbor meaningful? In: Beeri, C., Bruneman, P. (eds.) ICDT 1999. LNCS, vol. 1540, pp. 217-235. Springer, Heidelberg (1998)

2. Fritzke, B.: A growing neural gas network learns topologies. In: Advances in Neural Information Processing Systems 7, pp. 625-632. MIT Press (1995)

3. Holmström, J.: Growing Neural Gas Experiments with GNG, GNG with Utility and Supervised GNG. Master's thesis, Uppsala University (August 30, 2002)

4. Kohonen, T.: Self-Organization and Associative Memory, 3rd edn. Springer Series in Information Sciences, vol. 8. Springer, Heidelberg (1984)

5. Kohonen, T.: Self Organizing Maps, 3rd edn. Springer (2001) 
6. Martinetz, T.: Competitive hebbian learning rule forms perfectly topology preserving maps. In: Gielen, S., Kappen, B. (eds.) ICANN 1993, pp. 427-434. Springer, London (1993), http://dx.doi.org/10.1007/978-1-4471-2063-6_104

7. Martinetz, T., Schulten, K.: A "neural-gas" network learns topologies. Artificial Neural Networks 1, 397-402 (1991), http://web.cs.swarthmore.edu/ meeden/ DevelopmentalRobotics/fritzke95.pdf

8. Pacheco, P.: Parallel Programming with MPI, 1st edn. Morgan Kaufmann (1996)

9. Prudent, Y., Ennaji, A.: An incremental growing neural gas learns topologies. In: Proceedings of the 2005 IEEE International Joint Conference on Neural Networks, IJCNN 2005, vol. 2, pp. 1211-1216 (July 4-August 2005)

10. Sledge, I., Keller, J.: Growing neural gas for temporal clustering. In: 19th International Conference on Pattern Recognition, ICPR 2008, pp. 1-4 (2008)

11. Ultsch, A.: Clustering with SOM: U*C. In: Proc. Workshop on Self-Organizing Maps, Paris, France, pp. 75-82 (2005) 